\title{
Current Perspective of Traditional Chinese Medicines and Active Ingredients in the Therapy of Hepatocellular Carcinoma
}

\author{
Yuyao $\mathrm{Li}^{1, *}$, Yue $\mathrm{Li}^{1, *}$, Jinghao Zhang ${ }^{2}$, Longshan $\mathrm{ji}^{3}$, Man $\mathrm{Li}^{3}$, Xuehua Sun ${ }^{2}$, Hai Feng ${ }^{1}$, Zhuo Yu $\mathbb{D}^{2}$, \\ Yueqiu Gao ${ }^{1,2}$ \\ 'Institute of Infectious Disease, Shuguang Hospital Affiliated to Shanghai University of Traditional Chinese Medicine, Shanghai, People's Republic of \\ China; ${ }^{2}$ Department of Liver Disease, Shuguang Hospital Affiliated to Shanghai University of Traditional Chinese Medicine, Shanghai, People's Republic \\ of China; ${ }^{3}$ Laboratory of Cellular Immunity, Shuguang Hospital Affiliated to Shanghai University of Traditional Chinese Medicine, Shanghai, People's \\ Republic of China
}

*These authors contributed equally to this work

Correspondence: Zhuo Yu; Yueqiu Gao, Department of Liver Disease, Shuguang Hospital Affiliated to Shanghai University of Traditional Chinese Medicine, No. 528 Zhangheng Road, Pudong New District, Shanghai, 201203, People's Republic of China, Tel +86 2120256507 , Fax +86 21 20256699, Email zhuoyu@shutcm.edu.cn; gaoyueqiu@shutcm.edu.cn

\begin{abstract}
Hepatocellular carcinoma ( $\mathrm{HCC})$ is one of the leading lethal tumors worldwide, and the treatment remains a great medical challenge. Surgery and chemotherapy are current standard curative methods for patients with HCC, but the prognosis is still dismal. Based on unique medical theories and rich practical experience, traditional Chinese medicine (TCM) has been broadly employed to effectively treat HCC for a long history. Recently, systematic clinical trials have been well designed to study the efficacy of TCMs in the treatment of HCC, and the underlying antitumor mechanisms were also deeply explored. Here, we reviewed the published clinical evaluation of some commonly used TCMs in the treatment of HCC, and the related anti-HCC mechanisms through in vitro and in vivo study, promoting the modernization of TCM study in oncology for achieving a substantial reduction of HCC burden in the future.
\end{abstract}

Keywords: traditional Chinese medicine, hepatocellular carcinoma, antitumor mechanism, tumor therapy, active ingredient

\section{Introduction}

Hepatocellular carcinoma (HCC) ranks the sixth most common malignant neoplasm and the third cancer-caused death worldwide. ${ }^{1}$ Epidemiology demonstrated that the incidence of HCC is still rapidly increasing globally, especially in Europe and the United States. ${ }^{2}$ Although multiple noninvasive imaging-based surveillance avenues have been developed for the diagnosis of HCC, the beneficiary effect is limited by the uncertain presentation of HCC pathological changes in early stage. ${ }^{3}$ Surgical resection and liver transplantation are the current standard curative avenues for patients with regional HCC in early stage. However, the medical difficulties including high frequency of postoperative recurrence and the scarcity of donor liver resource seriously weaken the therapeutic efficacy on patients. ${ }^{4}$ Multi-kinase inhibitors including sorafenib and regorafenib have been approved to treat the unresectable or advanced HCC, but the improvement of patient survival quality is restricted due to drug resistance caused by the long-term administration. ${ }^{5}$ Therefore, other effective alternative therapeutic strategies with lower cost and fewer side effects are urgently required for the treatment of HCC.

Traditional Chinese medicine (TCM) has a long history for the treatment of cancer including HCC. The plethora of accumulated experience in the long-term therapeutic practice promotes the construction of unique medical theories of TCM. The holism of human body and the concept of treatment according to syndrome differentiation are the central foundation of these theories and become the vital principle of TCM in the diagnosis and treatment of cancer. In these theories, liver is the soul of the human body, functioning to store the blood, control the sinews, and maintain a smooth 
and uninterrupted flow of $q i .{ }^{6} \mathrm{HCC}$ is the consequence of systemic disease with causative cumulative toxicity, vital $q i$ deficiency and $q i$ flow stasis, leading to the disequilibrium of yin and yang. Therefore, the purpose of TCM treatment is to restore the balance of yin and yang in the body and keep the smooth flow of $q i$. The strategy of TCM in the treatment of HCC is to exert multiple methods according to different syndromes while treating the patients as a whole at the same time. Treatment methods, including nourishing yin, heat-clearing and detoxifying, invigorating blood and removing stasis, softening and resolving hard mass, and soothing liver $q i$ stagnation, are correspondingly used upon these TCM theories to effectively improve the overall survival of patients with HCC. ${ }^{7}$

Formula (Fufang) that integrates several herbal medicines according to syndrome differentiation is the main TCM prescription used in the therapy of disease. Although many effective formulas have been empirically proven to retard HCC progression and prevent HCC occurrence, the absence of scientific clinical evaluation hampers the standardization and modernization of TCM for the broad clinical application. Moreover, the complexity of innumerous ingredients in the formulas is also the obstacle to elucidate the antitumor mechanism of TCMs. Recently, as the important role of TCM in the treatment of HCC has been increasingly highlighted, well-designed clinical trials have been performed to evaluate the efficacy of several approved TCMs. ${ }^{8,9}$ In addition, the pharmacological action of TCMs and their active components has been investigated for understanding the antitumor mechanisms. In this review, we summarized the published clinical evaluation of some formulas in the treatment of HCC, and the exploration of related underlying anti-HCC mechanisms, promoting the modernization of TCM development.

\section{Clinical Evaluation of the Efficacy of TCMs}

As mentioned above, one of the major obstacles that hampers the modernization of TCMs is failing to identify pharmaceutical effects of the active component in the formulas, and few prescriptions have been put into scientifically rigorous clinical evaluation on their efficacy. Recently, this situation has gradually improved, and increasing clinical trials have been designed to evaluate the therapeutic effect of many TCMs by virtue of double blindness, control and randomization. Some traditional formulas and TCM products have been examined for their anti-HCC effect in clinical trials. Here, we review the clinical evaluation outcome of their pharmaceutical efficacy in the treatment of HCC (Table 1).

Table I Clinical Studies on the Efficacy of TCM in the Treatment of HCC

\begin{tabular}{|c|c|c|c|c|}
\hline Medication & $\begin{array}{l}\text { Patients } \\
\text { (Cases) }\end{array}$ & Enrolling Condition & Effect & Reference \\
\hline \multirow[t]{2}{*}{$\begin{array}{l}\text { Huaier } \\
\text { granule }\end{array}$} & 686 & $\begin{array}{l}\text { Patient underwent curative } \\
\text { resection of } \mathrm{HCC}\end{array}$ & Reduce recurrence rate and prolong recurrence-free survival & [14] \\
\hline & 62 & Patients diagnosed with $\mathrm{HCC}$ & $\begin{array}{l}\text { Prolong survival, reduce the number of interventional } \\
\text { treatment and improve the tumor response rate }\end{array}$ & [16] \\
\hline JPLQ & 150 & $\begin{array}{l}\text { Patients with unresectable HCC } \\
\text { who had received TACE treatment }\end{array}$ & $\begin{array}{l}\text { Relieve the Postembolization syndrome, improve the QoL of } \\
\text { patients after TACE, and repair liver function damage }\end{array}$ & [17] \\
\hline $\mathrm{CHHJ}$ & 125 & $\begin{array}{l}\text { Patients with unresectable HCC } \\
\text { who has received TACE treatment }\end{array}$ & $\begin{array}{l}\text { Improve 3-year overall survival and reduce the occurrence of } \\
\text { side effects after TACE }\end{array}$ & [19] \\
\hline$B X X X$ & 68 & Adults with advanced $\mathrm{HCC}$ & Achieve the median PFS of 6.07 months & [20] \\
\hline ESQJR & 137 & $\begin{array}{l}\text { Patients with } \mathrm{HCC} \text { underwent } \\
\text { tumor resection }\end{array}$ & Improve the prognosis and tumor recurrence & [2I] \\
\hline \multirow{2}{*}{$\begin{array}{l}\text { Jie Du } \\
\text { granule }\end{array}$} & 177 & Patients with advanced $\mathrm{HCC}$ & Prolong survival of patients with advanced $\mathrm{HCC}$ & [23] \\
\hline & 190 & HCC patients with PVTT & Prolong survival in HCC-PVTT patients & [24] \\
\hline
\end{tabular}

Abbreviations: HCC, hepatocellular carcinoma; TCM, traditional Chinese medicine; TACE, transcatheter arterial chemoembolization; JPLQ, Jian Pi Li Qi decoction; CHHJ, Chaihu Huaji decoction; BXXX, Banxia XieXin decoction; ESQJR, Erzhu Qinggan Jiedu Recipe; PFS, progression-free survival; QoL, quality of life. 
Huaier granule is the water-resolving products made of Huaier, trametes robiniophila murr, a genus of parasitic fungi growing on aging Chinese cassia trees. This TCM is approved to be used alone or combined with other drugs in the treatment of malignant tumors. Increasing studies demonstrate that the antitumor effect of Huaier granule may be associated with multiple biological activities, such as inhibition of cell proliferation, ${ }^{10}$ anti-metastasis,${ }^{11}$ interference of tumor angiogenesis ${ }^{12}$ and modulation of tumor-specific immune response. ${ }^{13}$ In recent years, more and more interest has been attracted to the role of Huaier granule in the treatment of HCC, and some clinical trials have been designed to evaluate the anti-HCC effect. A multicenter, randomized clinical trial enrolling 686 patients with HCC have proved the pharmacological activity of Huaier granule to effectively prolong recurrence-free survival by reducing intra- and extrahepatic recurrence after curative resection of HCC. ${ }^{14}$ The adjuvant therapy of TCMs for unresectable HCC upon transarterial chemoembolization (TACE) was investigated by a meta-analysis of randomized controlled trials. ${ }^{15}$ In the clinical trial conducted by Zhao et al, 62 patients with primary hepatic carcinoma were enrolled in the treatment using GSP-TACE combined with Huaier granule. ${ }^{16}$ The result of 42 month-lasting follow-ups showed the safety and efficacy of the combinatory regimen in the treatment of HCC.

Jian Pi Li Qi decoction (JPLQ) is one of the prescriptions of TCM that is commonly used to treat liver diseases in China. Documents evidenced that JPLQ could improve liver function and alleviate immune system impairment caused by toxins or chemical reagents. Further study showed that JPLQ could exert adjuvant function to enhance the anti-HCC effect in the combination of other antitumor remedies. A randomized, double-blind, placebo-controlled clinical trial was conducted in the cohort enrolling 150 patients with HCC who had received the therapy of TACE. ${ }^{17}$ Compared with control and placebo groups, JPLQ treatment could significantly relieve postembolization syndromes (PES) such as fever, pain, fatigue, lack of appetite, drowsiness, dry mouth, and constipation, and ameliorate the liver function damage caused by TACE as well. This outcome demonstrated that JPLQ decoction may serve as an effective modality to relieve PES and protect liver function to improve life quality of patients with HCC after TACE.

Chaihu Huaji decoction (CHHJ) is the prescription composed by Xiaochaihu-tang, an ancient classic Chinese herbal formula, and Huayu pill. The herbal medicine Xiaochaihu-tang is popular in the countries of East Asia and widely used in the treatment of hepatitis and liver cirrhosis with the activity of inhibiting inflammation and HCC progression. ${ }^{18}$ Huayu pill is able to mitigate blood stasis and reduce toxicity in liver disease. Accordingly, CHHJ has the synthetic pharmacological effect from both by integrating liver protection and blood flow improvement. In a retrospective study, a cohort of $125 \mathrm{HCC}$ patients who have received TACE treatment was recruited to investigate the complementary efficacy of CHHJ on the unresectable HCC. The result showed that CHHJ treatment could effectively alleviate the symptoms fever, nausea, vomiting, poor appetite, and leukocytopenia. In the follow-up, CHHJ could significantly prolong the 3-year overall survival probability in patients compared with control groups. ${ }^{19}$ This clinical trial supported that CHHJ has the ability to prevent adverse events after TACE and prolong overall survival of unresectable HCC patients when combined with TACE.

Banxia Xiexin decoction (BXXX) is originated from an old Chinese formula that was created by the famous doctor Zhang Zhongjing in Han dynasty. The first purpose of BXXX was to treat digestive diseases such as bloating and abdominal pain, and the usage has a long history of more than 1800 years. Recently, it has been found that BXXX could relieve the symptoms of patients with advanced liver cancer, inhibit tumor growth, and prolong survival in a retrospective study. Moreover, Wang et al conducted a clinical trial to evaluate the efficacy and safety of BXXX as monotherapy for patients with advanced HCC. ${ }^{20}$ In the study, BXXX monotherapy exhibited the median progression-free survival (PFS) of 6.07 months, whereas sorafenib, the first-line treatment of advanced HCC, displayed the median PFS of 2.8 months in China. These findings suggested that the efficacy of BXXX may be superior to that of sorafenib in patients with advanced HCC, although this was not a direct comparison. In addition, no drug-related serious adverse events were observed during the study. Therefore, BXXX is considered as a safe and effective alternative medicine for HCC treatment, and much precise and detailed study is further required.

Erzhu Qinggan Jiedu Recipe (ESQJR) is the prescribed formula of Chinese herbal medicine in Shuguang Hospital affiliated with Shanghai University of Traditional Chinese Medicine and has been used as a complementary regimen to treat patients with postoperative HCC for many years. Clinical observation showed that ESQJR treatment could reduce the HCC recurrent rate and prolong life survival. In a retrospective study, 137 patients who underwent HCC resection 
were enrolled and divided into two groups: 68 patients treated with western medicine and 69 patients treated with western medicine plus ESQJR. ${ }^{21}$ Compared with the western medicine group, the combinational treatment exhibited the reduction of cumulative recurrence rate, extension of overall survival and disease-free survival, and improvement of life quality and liver function of patients. Therefore, the clinical investigation confirmed the phenomenon that ESQJR functions as an adjuvant therapy to favor the rehabilitation of resected HCC patients.

Jie Du granule is the preparation of Chinese herbal medicine composed of Salvia chinensis Benth, root of Actinidia valvata, bulb of Cremastra appendiculata, and ventriculi galli mucosa. Many evidence in clinical application demonstrated that Jie du granule exerts cancer-fighting activity by inhibiting the proliferation of liver cancer. Among the ingredients, Salvia chinensis Benth and bulb of Cremastra appendiculata showed effective anti-angiogenesis and cytotoxic activity against Bel-7402 and SMMC-7721 HCC cells. ${ }^{22}$ Extracts of Actinidia valvata could inhibit tumor growth of transplanted $\mathrm{H} 22 \mathrm{HCC}$ cell in mouse model. Chen et al have performed a clinical retrospective study to evaluate the HCC-inhibitory efficacy of Jie du granule preparation alone. ${ }^{23}$ Compared to other best supportive treatments, Jie Du granule significantly prolonged the overall median survival of patients with advanced HCC. Besides the study of monotherapy, the complementary effect of Jie Du granule combined with other anti-HCC treatments was also evaluated in another retrospective research. ${ }^{24}$ The result indicated that Jie Du granule could increase the median overall survival from 11.3 months to 15.8 months for patients with advanced HCC who were treated with TACE plus gamma knife radiosurgery (GKR). Importantly, no adverse events occurred to induce death, suggesting that Jie Du granule acts as a safe and effective adjuvant alternative regimen to enhance the curative efficacy of HCC.

\section{The Mechanisms of TCM Underlying HCC Treatment}

Currently, the pharmacological mechanisms of TCM for treating HCC are continuously being explored in numerous studies. Multiple types of HCC cell lines in vitro and diverse animal models in vivo are used to discover the different anticancer mechanisms of medicines. Using these models, TCM has been studied in monotherapy in the form of active monomer, preparation and formula, or in combinational therapy with other avenues. Many experimental studies demonstrate that TCMs possess properties of targeting the tumor cells or modulating the tumor microenvironment through the regulation of a series of mutually interrelated biological pathways. Here, we elaborate the published mechanisms of TCM underlying HCC treatment in two aspects. One is the direct action on cancer cells to inhibit tumor growth through the cytotoxic effects. The other is the immunomodulation to stimulate the immune response to HCC.

\section{Targeting of Tumor Cells}

Direct killing tumor cells is one of the key functions of TCM on the HCC suppression, as evidenced by the short-term cell viability assay and long-term clonogenic assay. Further experimental studies demonstrate that TCM exhibits antiHCC activity through multiple ways, including proliferative inhibition, cell cycle arrest, apoptotic induction, pyroptosis, ferroptosis, autophagy, anti-oxidative effect, metastatic suppression, and anti-angiogenesis (Figure 1). Generally, TCM may function to affect one or more above mentioned process by targeting the signaling molecules.

\section{Inhibition of Cell Proliferation}

Cell proliferation is an important physiological process in organism that is regulated by multiple signaling molecules in cells. Various carcinogenic risk factors induce the dysregulation of these molecules, leading to abnormal uncontrolled cell proliferation and final tumorigenesis. It is evidenced that the mechanism of TCM to inhibit tumor growth is involved in the abrogation of abnormal molecular signaling pathway, restoring the balance between cell proliferation and death.

Bufalin is the major bioactive component of the TCM Chansu, and proven to have antitumor activity. Several studies demonstrated that bufalin could inhibit HCC cell proliferation in vitro and HCC growth in vivo by targeting the cell-cycle related kinase (CCRK). CCRK functions as an oncogene to promote cell proliferation by activating $\beta$-catenin/TCF signaling pathway. In HCC cells and tumor tissues, bufalin treatment could significantly downregulate CCRK expression, concomitant with the decrease of $\beta$-catenin and their downstream effectors CCND1 and EGFR. Therefore, bufalin suppresses HCC proliferation by inactivating CCRK-driven $\beta$-catenin/TCF signaling. ${ }^{25}$ Studies have shown that in male 


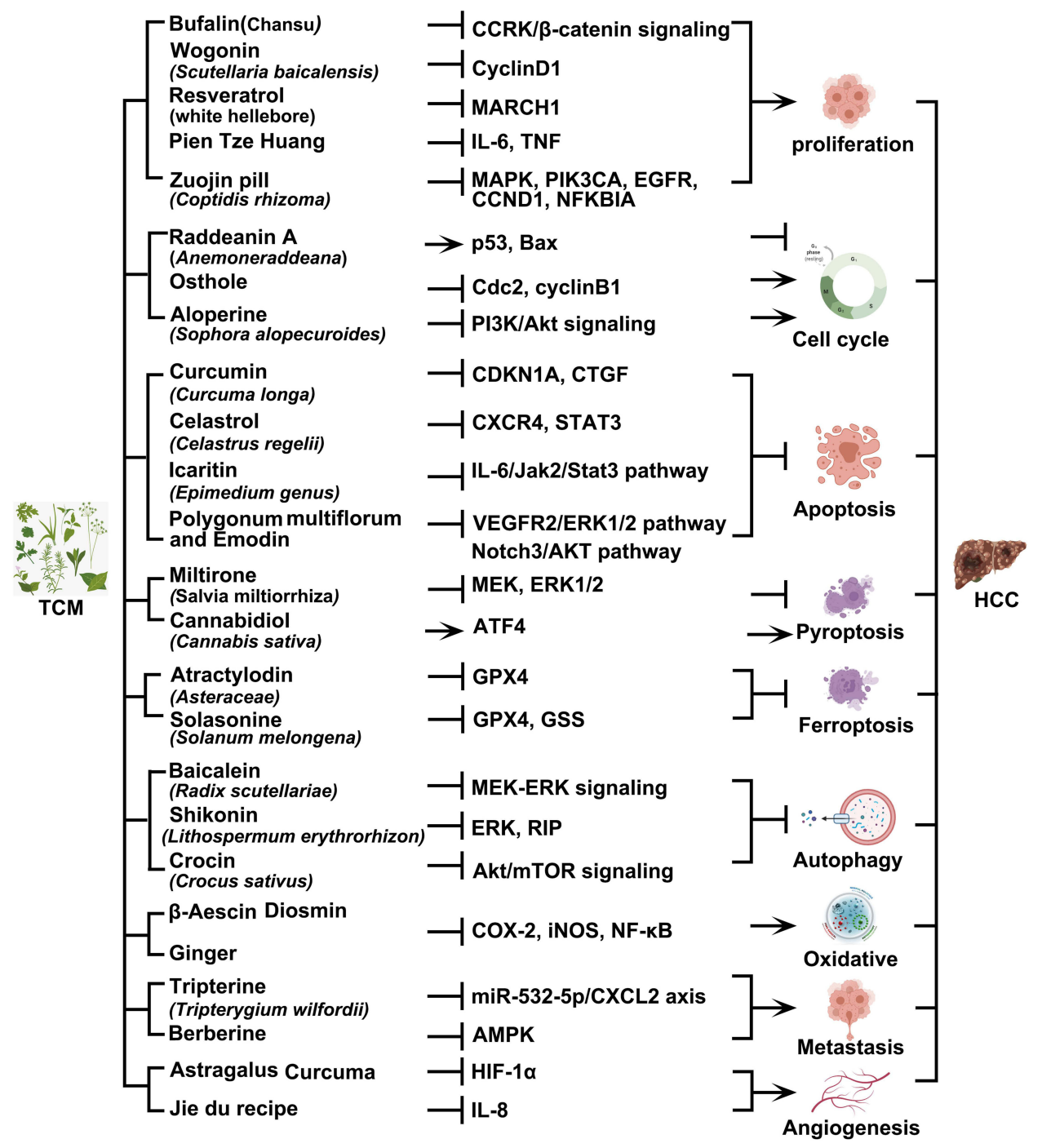

Figure I The inhibitory effect of Chinese herbal compound and monomers on HCC. Chinese herbal medicine inhibits the occurrence and development of HCC through multiple mechanisms such as inhibition of cell proliferation, cell cycle regulation, induction of apoptosis, inhibition of cells metastasis, induction of autophagy and inhibition of angiogenesis. Created with BioRender.com.

Abbreviations: HCC, Hepatocellular carcinoma; TCM, traditional Chinese medicine; CCRK, cell-cycle related kinase; GPX4, glutathione peroxidase 4.

HBV carriers, the activation of androgen receptor (AR) is closely related to the risk of HCC. Bufalin could effectively block HBV X protein-induced AR/CCRK/ $\beta$-catenin signaling, which subsequently led to cell cycle arrest and proliferative suppression in HBV-associated HCC cells. ${ }^{26}$

Wogonin is the natural ingredient isolated from Scutellaria baicalensis, and acts as a commonly used phytochemical with significant anti-carcinogenic and cancer-preventing activity. A study demonstrated that wogonin suppressed the proliferation of MHCC97L and HepG2 HCC cells by downregulating the expression of cyclinD1. ${ }^{27}$ In-depth study showed that wogonin-induced cyclinD1 proteolysis was associated with the activation of glycogen synthase kinase- 3 beta (GSK3 $\beta$ ). The site-directed mutagenesis assay validated that wogonin directly bound to GSK3 $\beta$ with high affinity. The result confirmed the molecular mechanism of wogonin to suppress HCC proliferation by targeting the regulation of GSK3 $\beta$ activity.

Pien Tze Huang (PZH) is an ancient well-known Chinese formula composed of Panax notoginseng, Moschus, Calculus bovis, and Snake gall. ${ }^{28}$ Studies demonstrated that PZH can treat various types of cancer and has exhibited promising therapeutic effects in clinical trials of HCC. According to the proteomics analysis of the tumor tissues from $\mathrm{HCC}$ xenograft Balb/c mice, PZH treatment can regulate 20 signaling pathways associated with inflammation, cancer 
growth and cell cycle. ${ }^{29}$ In the detection of protein expression, PZH decreased the expression of IL- 6 and TNF- $\alpha$ in the inflammation-related pathways, thus inhibiting inflammation and tumor growth. In addition, PZH also upregulated the phosphorylation of CCNB1 and FOXO3, and downregulated the phosphorylation of STAT3, leading to the decrease of cell number in S phase and the increase in G2/M phase. Therefore, PZH exhibited antitumor activity in HCC by targeting multiple signaling pathways involved in the inflammation-associated cytokine secretion, cancer growth pathways, and induction of $\mathrm{G} 2 / \mathrm{M}$ arrest.

Zuojin pill (ZJP) is a classic Chinese medicine formula composed of two herbs Coptidis rhizoma and Evodiae fructus. Alkaloids are proven to be the primary active compounds of ZJP and exhibit multiple pharmacological effects against cancer via targeting various signaling pathways. ${ }^{30}$ Using TCM pharmacological database and integrated database, a total of 224 compounds in ZJP were screened, among which 32 bioactive compounds were chosen to correspond to 86 targets in the compound-target analysis. Further analysis showed that MAPK1, PIK3CA, EGFR, CCND1, and NFKBIA were identified within these targets to be highly expressed in HCC cells and played an essential role in cell proliferation and survival. ZJP treatment could inhibit HCC cell proliferation and tumor growth, concurrent with the decrease of MAPK1, PIK3CA, EGFR, CCND1, and NFKBIA. These in vitro and in vivo experiments validated ZJP exerts prominent therapeutic effects on HCC mainly through the regulation of the EGFR/MAPK, PI3K/NF- $\mathrm{KB}$, and CCND1 signaling pathways. $^{31}$

Resveratrol is a natural product originally isolated from the roots of white hellebore and often used to treat liver disease. Several studies have evidenced that resveratrol exerts antitumor effect on HCC cells by inhibiting cell proliferation, invasion and migration. Zhang et al found that resveratrol treatment could upregulate p53 expression and downregulate Akt phosphorylation in MHCC97 HCC cells, leading to the suppression of cell proliferation through the induction of autophagy. ${ }^{32}$ In another study, MARCH1 was found to be a crucial oncogene to promote HCC progression of HepG2 and Hep3B cells via activating PTEN/Akt signaling. Resveratrol treatment could decrease the expression of MARCH1 and thus resulting in the inhibition of HCC proliferation, migration and invasion. ${ }^{33}$

\section{Cell Cycle Regulation}

Cell cycle is an important regulatory pattern of cell proliferation and differentiation involved in physiological processes such as embryonic generation, tissue formation and organ development. It is precisely regulated by a network composed of some key molecules, so-called checkpoints, including cell-cycle dependent kinase and cyclin. ${ }^{34}$ Once the abnormality occurs in any link of the network, it causes cell cycle disorder and uncontrolled cell growth. Evidence demonstrates that cancer is a type of uncontrolled growth, and the disorder of cell cycle promotes the excessive proliferation of tumor cells including HCC. These aberrant signaling molecules provide a potential source of targets for cancer therapy.

Raddeanin A (RA) is one of the major active monomers extracted from Anemone raddeana regel. It was reported that RA holds antitumor function by prohibiting proliferation, invasion, migration, adhesion of tumor cells. A study regarding the combinational therapy showed that RA exerted synergistic effect on cisplatin in the treatment of high-risk metastatic QGY-7703 HCC cell by activating p53 and Bax. ${ }^{35}$ The upregulation of p53 and Bax increases the proportion of tumor cells in G0/G1 phase and decreases those molecules in $\mathrm{S}$ phase. This induction of cell cycle arrest caused by RA strengthens the proliferative inhibition of cisplatin.

Osthole, a bioactive coumarin derivative, is a Chinese herbal medicine that is reported to exhibit various pharmacological functions. Previous studies have shown osthole possess hepatocellular protective properties and antitumor activity. In the research of TCM against HCC, Lin et al found that osthole could inhibit cell proliferation and migration in SMCC-7721, MHCC-97H, HCC-LM3 and BEL-7402 HCC cells. ${ }^{36}$ Cell cycle checkpoints Cdc2 and cyclinB1 were found to be highly expressed in these HCC cells, while osthole treatment could downregulate their expression. Targeting $\mathrm{Cdc} 2$ and cyclinB induced cell cycle arrest of HCC cells in G2/M phase, leading to the suppression of HCC proliferation.

Aloperine (ALO) is a quinolizidine alkaloid extracted from Sophora alopecuroides, which exerts anti-neoplastic effect on a variety of cancer cells including HCC. Researches demonstrated that activation of PI3K/Akt signaling pathway was associated with abnormal cell cycle progression and cell proliferation in HCC. ALO was found to inhibit the activity of PI3K/Akt signaling by decreasing the level of p110 $\alpha$, p85, Akt and p-Akt in Hep3B and Huh7 HCC cells, 
thus leading to cell cycle G2/M phase arrest. ${ }^{37}$ Moreover, inhibition of Akt by siRNA augmented the effect of ALO on the suppression of HCC proliferation and growth in vitro and in vivo, further supporting the mechanism of ALO to inhibit HCC proliferation through the induction of cell cycle arrest.

\section{Induction of Apoptosis}

Apoptosis, known as programmed cell death, is defined as cellular self-restricted regulation in the process of development and aging to keep dynamic balance of cell in physiological condition. ${ }^{38}$ Multiple events such as cellular stress, DNA damage and immune surveillance could induce apoptosis through the regulation of several intrinsic and extrinsic signaling pathways. In tumorigenesis, apoptosis acts as a defensive avenue to protect the tissues by eliminating deleterious cells in the lesion. Cancer cells tend to develop the ability to evade the induction of apoptosis, thus resisting the remedy of therapeutic agents. ${ }^{39}$ Therefore, it is one of the important antitumor mechanisms of TCM to induce cancer cell death via targeting the apoptotic-related signaling.

Curcumin is the principal polyphenolic curcuminoid isolated from the plant Curcuma longa ${ }^{40}$ It has been reported that curcumin treats HCC in terms of induction of apoptosis. An analysis of different gene expression indicated that curcumin inhibits cell proliferation of PLC5 HCC cells by downregulating TGF- $\beta$ signaling, and CDKN1A and CTGF are proved to be the key targets of curcumin in the TGF- $\beta$ signaling. Inhibition of CDKN1A and CTGF by curcumin induced apoptosis in HCC cells, leading to the suppression of tumor growth. ${ }^{41}$

Celastrol is the plant compound extracted from the roots of Celastrus regelii, which exerts beneficial effects on a variety of cancers in clinical trials. In a study, chemokine CXCR4 was identified as the target of celastron in the treatment of HCC. CXCR4 could promote cell proliferation and apoptotic suppression through the upregulation of PI3K/ Akt signaling. Celastrol treatment inhibited the activity of PI3K/Akt signaling via reducing the expression of CXCR4, thus inducing apoptosis and tumor suppression in HCC. ${ }^{42}$ In addition, STAT3 was also proved to be another target of celastrol for the induction of apoptosis. Celastrol blocked the activity of STAT3/JAK2 signaling by directly inhibiting STAT3, leading to the suppression of tumor growth in the xenograft HCC mouse. ${ }^{43}$

Icaritin is a prenylflavonoid derivative from Epimedium genus that has been used as an anticancer agent for various cancers including HCC. Icaritin treatment could upregulate the cleavage of caspase 3, the marker of apoptosis, in PLC5 and Huh7 HCC cells. In addition to the caspase-dependent pathway, icaritin was also confirmed to induce apoptosis through the regulation of IL-6/Jak2/Stat3 pathway. ${ }^{44}$ Moreover, icaritin provoked the immunogenic cell death associated with apoptosis and mitophagy, leading to the immune response to $\mathrm{HCC} .{ }^{45}$

Polygonum multiflorum (PMT) is a member of Polygonaceae, and the tuberous root is the main medicinal part of TCM used to constitute many formulas such as Jiangzhining and Yiganning granules. ${ }^{46}$ PMT is usually processed before prescription preparation, and processed PMT displays the property of liver tonification. Evidence demonstrated that processed PMT exerts anti-HCC effects by inhibiting cell proliferation and invasion and promoting apoptosis. At present, 133 chemical components have been isolated from processed PMT, which contain anthraquinones, lecithin, polysaccharides and tannins. ${ }^{47}$ Emodin, as one of the major components extracted from processed PMT, induces tumor cell death by regulating multiple signaling pathways. It has been reported that emodin initiates apoptotic pathway by inhibiting PI3K/AKT and ERK signal transduction. Another study indicated that emodin shows the ability to increase HCC sensitivity to Fas and TRAIL-mediated apoptosis by inhibiting casein kinase 2 (CK2). ${ }^{48}$ Moreover, emodin inhibits the activity of VEGFR2/ERK1/2 pathway by promoting miR-34a-mediated signal transduction, thus blocking the migration and invasion of MHCC-97H HCC cells. ${ }^{49}$ Physcion is another crucial component of processed PMT, which could inhibit cell proliferation of Huh7 and Bel-7402 HCC cells through the promotion of AMPK phosphorylation and Caspase family-mediated apoptosis pathway. ${ }^{50}$ In addition, physcion could inhibit Notch3 and p-AKT expressions via regulating Notch3/AKT pathway, leading to the reduction of liver cancer cells and reversal of drug resistance to sorafenib. ${ }^{51}$ Therefore, PMT has been confirmed to be a potent antitumor TCM in the treatment of HCC through multiple pharmacological mechanisms.

\section{Induction of Pyroptosis}

Pyroptosis, also known as cellular inflammatory necrosis, is a new form of programmed cell death characterized by the activation of a strong inflammatory response via the production of danger-associated signaling molecules and 
cytokines. ${ }^{52}$ Gasdermin D (GSDMD) and gasdermin E (GSDME), the members of gasdermin family, function as the hallmark for the provocation of pyroptosis through the proteolytic fragmentation catalyzed by caspase 1/4/5/11 and caspase 3, respectively. ${ }^{52,53}$ Recently, some studies found that the expression profile of pyroptosis-related molecules can reflect the pathological status of cancer. ${ }^{54}$ Thus, the regulation of pyroptosis is a promising avenue for TCMs and their active compounds to suppress HCC development.

Miltirone is a derivative of phenanthrene-quinone isolated from the root of Salvia miltiorrhiza Bunge, which has been found to possess antitumor activity. ${ }^{55}$ A study conducted by Zhang et al demonstrated that miltirone could suppress phosphorylation of mitogen-activated and extracellular signal-regulated kinase (MEK) and extracellular regulated protein kinases 1/2 (ERK1/2), leading to the reduction of cell viability of HeG2 and Hepa1-6 HCC cells. This effect was associated with the caspase 3-mediated proteolytic cleavage of GSDME, and knockdown of GSDME could switch miltirone-induced cell death from pyroptosis to apoptosis. Therefore, miltirone was proved to be a potential therapeutic agent for the treatment of HCC via GSDME-mediated pyroptosis.

Cannabidiol (CBD), a phytochemical derived from Cannabis sativa, has been shown to display the antitumor property in a variety of cancers. ${ }^{56}$ Increasing studies demonstrated that CBD and other cannabinoids could function to induce cell growth arrest, retard cell metastasis and stimulate cell apoptosis, resulting in the suppression of tumor progression. ${ }^{57}$ Recent study has evidenced that CBD effectively suppressed HCC cell growth in vivo and in vitro, while GSDME knockdown could counteract such antitumor effects. This result suggested the association of antitumor activity of CBD with the provocation of pyrotosis. Moreover, further research indicated that CBD induced integrative stress response (ISR)-dependent ATF4 activation, which contributes to the promotion of GSDME-dependent pyroptosis.

\section{Induction of Ferroptosis}

Ferroptosis is a form of regulated cell death driven by iron-dependent lipid peroxidation, which is morphologically, biologically, and genetically distinct from necrosis and autophagy. ${ }^{58}$ Phospholipids are the components of lipid bilayers that make up cellular membrane, and peroxidation of phospholipids is the key driver of ferroptotic death. ${ }^{59}$ Researches indicated that ferroptosis could be triggered by diverse physiological conditions and pathological stresses. ${ }^{60}$ It has been reported that ferroptosis plays a pivotal role in the depression of tumorigenesis by removing the deficient or damaged cells in the environment. ${ }^{61}$ Therefore, the fast-growing studies of ferroptosis in cancer have boosted a perspective for cancer therapy. ${ }^{62}$

Atractylodin is the major active component of atractylode, the plant of Asteraceae, which has been reported to show inhibitory function on tumor development and progression. Studies indicated that atractylodin has the ability to induce apoptosis of cholangiocarcinoma via the activation of caspase cascade and inflammation and to provoke oxidative stress and phospholipids peroxidation as well. ${ }^{63}$ Moreover, glutathione peroxidase 4 (GPX4) acts as the key regulator of lipid peroxidation and iron metabolism, which is involved in the regulation of ferroptosis. ${ }^{59}$ In HCC cells, atractylodin was observed to induce the accumulation of lipid peroxides and ferroptosis by inhibiting GPX4 expression, leading to the suppression of HCC progression. ${ }^{64}$ Therefore, it is suggested that ferroptosis plays a significant role in the inhibitory effect of atractylodin on HCC cells.

Solasonine, the natural glycoalkaloid aglycone isolated from Solanum melongena, exhibits the activity to inhibit the proliferation of various tumor cells. ${ }^{65,66}$ Some studies demonstrated solasonine suppressed HCC cell proliferation and invasion via $\mathrm{G} 2 / \mathrm{M}$ phase cell cycle arrest. Moreover, the fact that solasonine was able to induce lipid oxidative stress to cause the inflammatory events that triggered ferroptosis was found in the treatment of HepG2 HCC cells. ${ }^{67}$ Administration of ferrostatin-1, the ferroptosis inhibitor, significantly downregulated solasonine-induced oxidative stress, leading to the reversal of HCC suppression. Furthermore, the expression levels of GPX4 and GSS were reduced in response to the induction of ferroptosis, suggesting solasonine tunes ferroptosis by targeting GPX4 and GSS. Thus, solasonine participates in ferroptosis in the treatment of HCC, which facilitate further development of antitumor therapy.

\section{Induction of Autophagy}

Autophagy is a conserved tightly orchestrated biological process to clear damaged or aged cellular components for the purpose of cellular homeostasis. The bulk of misfolded proteins, impaired organelles, or intracellular pathogens are 
coated into a double-membranous vesicle termed as autophagosome, which is fused with lysosomes to form autophagic lysosome and ultimately removed through the fragmentation and degradation. Aberrations in autophagy result in various pathological states including cancer. Generally, autophagy is thought to be the cause of survival, but over-activation of autophagy may lead to a non-apoptotic cell death. Therefore, manipulation of autophagy as a potential tumor suppressor by regulating autophagic signaling is a promising therapeutic regimen in the treatment of cancer including HCC.

Baicalein is the principal bioactive monomer isolated from Radix scutellariae and exhibits antitumor effects in vitro and in vivo. Previous studies showed that baicalein treatment inhibited the lung metastasis of HCC orthotopically transplanted in athymic mice. This effect was associated with the induction of autophagy occurring in HCC cells, as evidenced by the reduction of mitochondrial transmembrane potential and activation of caspase- 9 and caspase- $3 .{ }^{68}$ Moreover, baicalein also exerted cytotoxicity and induced autophagy by inhibiting MEK-ERK signaling pathway when combined with Paclitaxel. ${ }^{69}$

Shikonin is a type of naphthoquinone extracted from Lithospermum erythrorhizon, and shows potential anticancer activity as a cancer therapeutic agent. It has been reported that shikonin has the ability to induce apoptotic cell death or trigger cell cycle arrest in various cancers by regulating molecules in Bcl-2 family and NF- $\mathrm{kB}$ signaling pathway. ${ }^{70,71}$ Recent studies demonstrated that LC3-II expression, the marker of autophagy, was increased in the HCC cells treated with shikonin. The activity of Ras/Raf/MEK/ERK pathway was connected with the occurrence of autophagy, and knockdown of ERK could counteract the effect of shikonin on increasing LC3-II accumulation. In addition to the targeting of ERK, shikonin was also found to enhance autophagy through the suppression of RIP. In Huh7 xenograft mouse model, shikonin was confirmed to inhibit tumor growth by initiating autophagy through the downregulation of ERK and RIP. ${ }^{72}$

Crocin is the main component of saffron originated from the Chinese herbal Crocus sativus. Crocin displays protective roles in HCC from early stage to late stage by targeting various downstream effectors in the signaling pathways. ${ }^{73}$ A study showed that crocin treatment in HCC gave rise to BECN1 upregulation, LC3-II accumulation, and p62 decrease, indicating the occurrence of autophagy in HCC cells. Akt/mTOR signaling plays an important role in autophagy regulation of cancer cells. It was evidenced that crocin induces autophagy by inhibiting the activity of Akt/ mTOR signaling through the downregulation of $\mathrm{p}$-Akt and $\mathrm{p}-\mathrm{mTOR}{ }^{74}$

\section{Anti-Oxidative Effect}

Oxidative stress (OS) is a well-recognized biological event that plays a prominent role in the progression of HCC. Abnormal OS is responsible for the increased level of reactive oxygen species (ROS) that are noxious to cell by causing mutation and uncontrolled cell division. Additionally, they also contribute to the regulation of onco-suppressor, oncopromoter, and several pro-inflammatory genes involved in the development of HCC. Some TCMs possess the antioxidative activity, which exhibit the protective effect against liver cancer by reducing OS condition. ${ }^{75}$

$\beta$-Aescin (AES) is a triterpene saponin derived from the horse chestnut tree Aesculus hippocastanum with the antioxidative activity against liver damage. ${ }^{76}$ Diosmin (DIO) is an unsaturated flavonoid glycoside extracted from citrus fruits, exhibiting protective effects on liver injury in animal models. ${ }^{77}$ The combination of AES and DIO was found to alleviate liver damage in CCl4-induced rats in a synergistic manner. ${ }^{78}$ Ginger is one of the most common traditional herbal medicines, and its biological active compounds, gingerol and shogaol, possess anti-oxidant activity with hepatoprotective effects. ${ }^{79}$ In vivo study indicated that ginger and its extract gingerol promoted a strong protective effect against DEN-induced hepatocarcinogenesis in rat. This effect was confirmed to be related to the prevention of oxidative stress by reducing COX-2, iNOS and NF- $\mathrm{KB}$ expression. ${ }^{80}$

\section{Inhibition of Cells Metastasis}

Malignant cell invasion and migration is one of the main causes of death in patients with HCC. Tumor migration and invasion is a complex, multi-step, multi-factor process involved in the regulation of many signaling pathways. Hence, targeting these signaling molecules to interfere with tumor metastasis is one of the therapeutic strategies of TCM in the treatment of HCC. 
Tripterine is a natural compound mainly derived from Tripterygium wilfordii with the character of anticancer activity. Some microRNAs (miRNAs) play crucial roles in modulating cancer cell growth and metastasis. miR-532-5p was reported to be downregulated in HCC cells, while tripterine treatment could significantly increase miR-532-5p to induce the suppression of HCC metastasis. Moreover, the inhibition of HCC metastasis caused by tripterine was also dependent on the decrease of CXCL2. The result indicates tripterine functions as the miR-532-5p/CXCL2 axis inhibitor to block HCC tumorigenesis and metastasis. ${ }^{81}$

Berberine, an alkaloid extracted from herbal plants, was evidenced to deploy anticancer effects on various cancers. $\beta$ catenin was identified as a pivotal molecule to provoke HCC metastasis by tuning pro-oncogenic signaling pathways. Berberine was found to repress $\beta$-catenin translation in Hep3B and HepG2 HCC cells, thus inhibiting cell migration and invasion. Moreover, mTOR activity was also decreased upon berberine treatment, which was associated with suppression of HCC metastasis through the blockage of $\beta$-catenin signaling. ${ }^{82}$ AMPK was a $\beta$-catenin-independent target of berberine in HCC cells, and anti-metastatic effect of berberine was found to link with the activation of AMPK. ${ }^{83}$

\section{Inhibition of Angiogenesis}

Abnormality of angiogenesis in tumor tissues is the prominent character of HCC. Abundant vascular construction such as arterialization and sinusoidal capillarization facilitates cancer cells to loot nutrients for substantial outgrowth, and to disseminate with the blood flow to the distant place or other organs. Dysregulation of angiogenic signaling pathways has been identified as the cause of abnormal angiogenesis in cancer, and plays a fundamental role in the proliferation, invasion and metastasis of HCC. Many growth factors or cytokines, which function as the pivotal regulators in these angiogenic pathways, are the potent targets of TCM that are practically selected and validated for the therapy of HCC.

VEGF and its receptors VEGFR are the well-researched effectors of angiogenesis, which could be regulated by other factors in the angiogenic signaling pathways. IL-8 has been evidenced to trigger angiogenesis by stimulating the expression of VEGF and VEGFR through the activation of NF- $\mathrm{KB}$ signaling in $\mathrm{HCC}{ }^{84} \mathrm{Jie}$ du recipe inhibited HCC proliferation in clinical trial, concomitant with the decrease of VEGF level in tumor tissues. This anti-angiogentic effect caused by Jie du recipe was evidenced to be associated with IL-8 suppression, as activation of AKT and ERK in the downstream of NF- $\mathrm{KB}$ signaling pathway could reverse the reduction of VEGF and VEGFR expression. Jie du recipe also prohibited HIF-1 $\alpha$ expression, another important factor to upregulate VEGF level, thus exhibiting anti-angiogenic effect and growth suppression in HCC. ${ }^{85}$ Astragalus membranaceus and Curcuma wenyujin are widely prescribed Chinese herbs for cancer therapy in the clinical practice. The combination of both drugs promoted vascular normalization in tumor-derived endothelial cells of HCC by increasing CD34 expression and reducing HIF-1 $\alpha$ expression. ${ }^{86}$

\section{Regulation of Immune Cells}

Immunotherapy demonstrated marvelous curative efficiency as an alternative therapeutic regimen in the treatment of advanced cancers. The immunosuppressive microenvironmental specificity inherent in liver restrains the medical efficacy on HCC by facilitating immune evasion of cancer cells. TCM has been evidenced to show immunoregulatory properties of restoring immunosurveillance and enhancing immune response to HCC. Through the upregulation of immunostimulatory factors or downregulation of immunosuppressive factors, TCM acts on multiple targets in several ways to exert antitumor effect. Here, we review the immunoregulatory potency of individual TCM or formulas on the treatment of HCC via controlling various immune cells and their related immune signals in the remodeling of immune microenvironment, providing new insights on the mechanisms of TCM underlying immunotherapy on HCC (Figure 2).

\section{Activation of Stimulatory Immune Cells}

In addition to cancer cells, the abundant immune cells are filled in the TME involved in tumor proliferation and metastasis. According to the cell differentiation and function, immune cells in the TME of HCC can be classified into two groups: immunostimulatory lymphocytes, and immunosuppressive myeloid cells and lymphocytes. The representative stimulatory immune cells include cytotoxic $\mathrm{CD} 8^{+} \mathrm{T}$ cells, $\mathrm{CD} 4^{+} \mathrm{T}$ cells with helper 1 type, and natural killer (NK) cells. NK cells and $\mathrm{CD}^{+} \mathrm{T}$ lymphocytes are, respectively, known to be the primary antitumor effectors in innate and adaptive immune response, and $\mathrm{CD}^{+} \mathrm{T}$ helper 1 cells exert the adjuvant function on stimulating the tumor-killing 


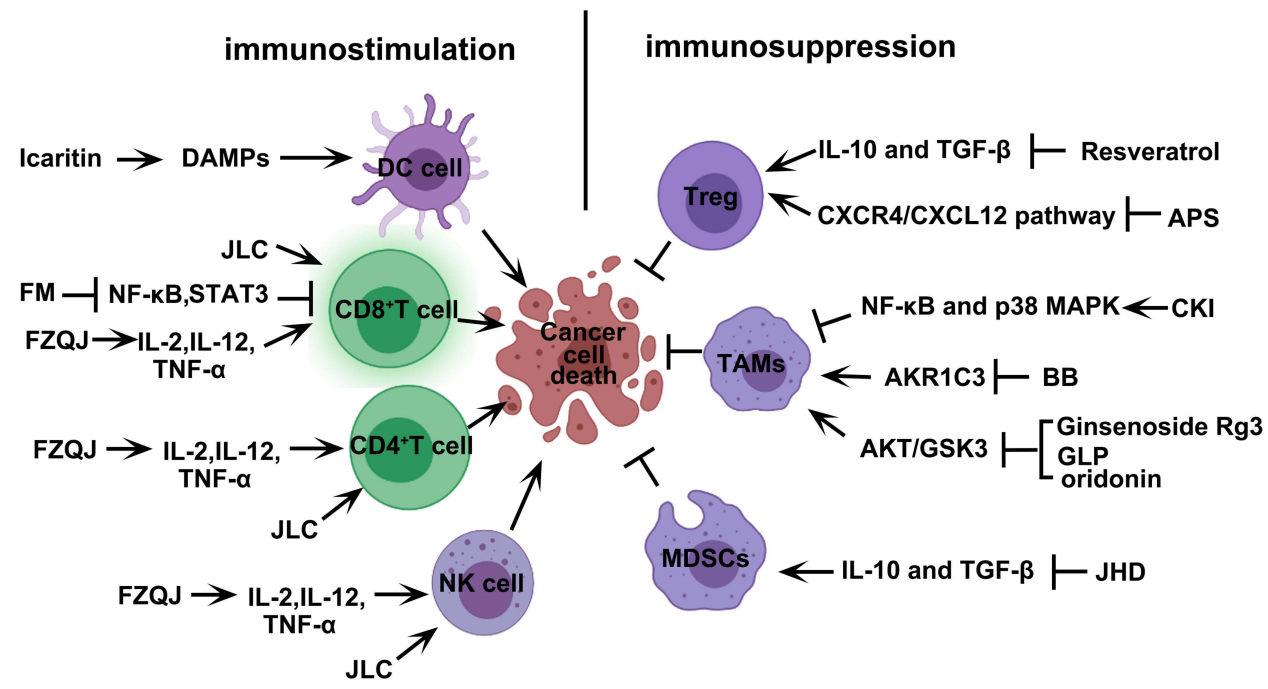

Figure 2 TCM induces cell death in HCC through regulation of various immune cells in the TME. TCM including compound and monomers enhances the immune response by inducing the activation of immunostimulatory cells $\left(\mathrm{CD} 8^{+} \mathrm{T}\right.$ cell/ $\mathrm{CD} 4^{+} \mathrm{T}$ cell/DC cell/NK cell) and suppressing immunosuppressive cells (Treg/ MDSCs/ TAMs). Created with BioRender.com.

Abbreviations: DAMPs, damage-associated molecular patterns; DC, dendritic cell; ICD, immunogenic cell death; JLC, Jinlong capsule; FZQJ, Fuzheng Qingjie Granules; FM, Frankincense and myrrh; Treg, Regulatory T; MDSCs, myeloid-derived suppressor cells; TAMs, tumor-associated macrophages; APS, Astragalus polysaccharides; JHD, jianpi huayu decoction; CKI, Compound Kushen Injection; BB, Bombyx batryticatus; GLP, ganoderma lucidum polysaccharide.

effectors in immune response and immune memory. Activation of stimulatory immune cells to promote the release of antitumor proinflammatory cytokines is one of the mechanisms that TCM inhibits tumor growth in HCC immunotherapy.

Icaritin has been reported to prohibit cell proliferation in HCC through apoptotic induction. Additionally, icaritin caused the dying tumor cells to release the damage-associated molecular patterns (DAMPs), which could be recognized and processed by dendritic cells (DCs). Subsequently, DCs presented the immunogenic portion of the processed DAMP to $\mathrm{CD}^{+} \mathrm{T}$ cells, and activated them to convert into cytotoxic $\mathrm{T}$ effectors. Cytotoxic $\mathrm{CD} 8^{+} \mathrm{T}$ cells attacked cancer cells via secretion of perforin, granzyme A and granzyme B. The whole process is called immunogenic cell death (ICD). Importantly, the chemotherapeutic drug doxorubicin also showed the activity of ICD, and the combination with icartin could synergistically enhance the T cell-mediated immune response to HCC. ${ }^{45}$

Jinlong capsule (JLC), composed of three medicinal animals Bungarus, Agkistrodon and Gecko, significantly induced the T cell immune response to $\mathrm{HCC}$ by increasing the proportion of cytotoxic $\mathrm{CD} 8^{+}$effector T cells. In consistency to the increase of $\mathrm{CD}^{+} \mathrm{T}$ cells, JLC also promotes the accumulation of $\mathrm{CD}^{+} \mathrm{T}$ cell and NK cells in the tumor tissues of HCC. The complete activation of various stimulatory immune cells reflects the immunomodulatory characteristics of JLC as a potent therapeutic agent in the anti-HCC treatment. ${ }^{87}$

Fuzheng Qingjie (FZQJ) granules are composed of four Fuzheng Guben herbs including Astragalus membranaceus, Ligustrum lucidum, Ganoderma lucidum and Rhizoma dioscorea, and two Qingre Jiedu herbs including Hedyotis diffusa and Prunella vulgaris. It has been reported that FZQJ granules exert the adjuvant effect in the treatment of HCC. A study showed that FZQJ granules could increase the level of $\mathrm{CD}^{+} \mathrm{T}$ helper cells when suppressing tumor growth in the HCCbearing mouse model. The tumor-inhibitory effect of FZQJ granules was found to be impaired by the depletion of CD4 $\mathrm{T}$ cells. The accumulation of $\mathrm{CD}^{+} \mathrm{T}$ cells promoted the production of pro-inflammatory cytokines such as IL-2, IL-12 and TNF- $\alpha$, and the subsequent activation of $\mathrm{CD}^{+}$T cells and NK cells. Thus, FZQJ granules stimulated T cell-mediated immune response to $\mathrm{HCC}$ via the recruitment of $\mathrm{CD}^{+}{ }^{\mathrm{T}}$ helper cells. ${ }^{88}$

Frankincense and myrrh (FM) are traditional herbal drugs with the anti-inflammatory activity, and both are usually combined to achieve a synergistic effect in clinical practice. ${ }^{89} \mathrm{HCC}$ has been considered as a type of inflammation-related cancer, and thereby FM has recently been used in the treatment of HCC via regulating inflammatory immune cells in the TME. It was evidenced that constitutive activation of NF- $\kappa$ B and STAT3 caused immune suppression on $\mathrm{CD} 8^{+} \mathrm{T}$ cells responsible for 
antitumor immunity. FM could restore $\mathrm{CD}^{+} \mathrm{T}$ cell-mediated antitumor activity via downregulating NF- $\mathrm{KB}$ and STAT3 in HCC. The increased production of IFN- $\gamma$ within TME further confirmed the ability of FM to stimulate $\mathrm{CD} 8^{+} \mathrm{T}_{\text {cells. }}{ }^{90}$

\section{Inhibition of Suppressive Immune Cells}

Apart from stimulatory immune cells, suppressive immune cells exist in the TME, and especially, predominant in terms of quantity and activity to maintain the immunosuppressive specificity for the survival of HCC. Regulatory $\mathrm{T}$ (Treg) cells, myeloid-derived suppressor cells (MDSCs) and tumor-associated macrophages (TAMs) are the main suppressive immune cells that contribute to the building of immunosuppressive microenvironment. These cells release various protumor inflammatory cytokines not only to promote cancer cell proliferation, but also to suppress the activity of stimulatory immune cells. In some cases, TCMs exhibit antitumor activity by inhibiting the activation of suppressive immune cells and the release of tumor-promoting cytokines.

Treg cells are one of the key suppressive immune cells prevailing in the HCC microenvironment. Resveratrol, originated from the root of white hellebore, could inhibit tumor growth in the HCC-bearing mouse model by decreasing the number of Treg cells. The subtype of $\mathrm{CD} 8^{+} \mathrm{CD} 122^{+}$Tregs was confirmed as the target of resveratrol, as the transfer of $\mathrm{CD}^{+} \mathrm{CD} 122^{+}$Tregs into the mice reversed the antitumor effect. Concomitant with the decrease of Tregs, resveratrol downregulated the immunosuppressive cytokines TGF- $\beta$ and IL-10, and upregulated immunostimulatory antitumor cytokines TNF- $\alpha$ and IFN- $\gamma$. The production of TNF- $\alpha$ and IFN- $\gamma$ improved the immunosuppressive microenvironment and enhanced antitumor $\mathrm{T}$ cell immune response. In addition, resveratrol recruited IFN- $\gamma$-expressing $\mathrm{CD} 8^{+}$effector $\mathrm{T}$ cells, resulting in the cytotoxic effect on HCC cells. ${ }^{91}$ Astragalus polysaccharides (APS), a traditional Chinese medicinal herb extracted from Astragalus membranaceus, could restore the cytokine imbalance and block SDF-1 or its receptor to inhibit migration of Treg cell by regulating CXCR4/CXCL12 pathway. ${ }^{92}$

MDSCs are a group of immature suppressive immune cells contributing to the building of the HCC immunosuppressive microenvironment. Previous studies showed that jianpi huayu decoction (JHD) suppressed the expression of IL-10 and TGF- $\beta$ to reduce HCC cell viability. The downregulation of immunosuppressive cytokines was associated with decrease of MDSCs infiltration in the JHD-treated HCC cells. STAT3 was identified as the target of JHD in the manipulation of MDSCs, whose expression could counteract the inhibitory effect of JHD on the differentiation of MDSCs. JHD also inhibits the activity of iNOS, thus neutralizing the immunosuppressive ability of MDSCs by reducing the endogenous level of ROS. Moreover, the suppression of MDSCs caused by JHD could relieve the inhibitory effect on $\mathrm{CD}^{+}$T cell activation in the H22 HCC-bearing mice. ${ }^{93}$

TAM plays an important role in repressing the antitumor activity of $\mathrm{T}$ cell or other immunostimulatory cells. A large number of studies have shown that TAMs are typical pro-tumor macrophages, which are responsible for the release of immunosuppressive cytokines. Compound Kushen Injection (CKI), an approved traditional Chinese medicine formula, is extracted from the roots of Kushen and Baituling. In general, macrophages can be polarized into classically activated M1 and alternatively activated M2. The combination between CKI and low-dose sorafenib could switch M2 to M1 status and relieve immunosuppression by triggering TNFR1-mediated NF- $\mathrm{KB}$ and $\mathrm{p} 38$ MAPK signaling cascades in the HCC microenvironment. ${ }^{94}$ Bombyx batryticatus (BB) is one of the animal-TCMs frequently used as anticancer agent in HCC treatment. A study showed that BB functioned to regulate the differentiation of macrophages in patients with viral-associated HCC and non-viral-associated HCC. By inhibiting the expression of AKR1C3, BB reduced the recruitment of TAMs in the HCC microenvironment, thus improving the prognosis and survival rate of patients. ${ }^{95}$ Ginsenoside Rg3, ganoderma lucidum polysaccharide (GLP), and oridonin as the combined therapy could restore immune function by reducing the proportion of M2-polarized macrophages and inhibit the p-EGFR by regulating AKT/GSK3 signaling pathways. ${ }^{96}$

\section{Conclusion}

The occurrence of HCC is related to multiple risk factors. One of the epidemiological features of HCC is the high mortality rate in patients, partly because the early symptoms are unobvious, and when it is diagnosed, it is mostly in the middle and late stages. At present, surgery, radiotherapy and chemotherapy are often used, but the malignant disease is still not completely cured, and the life quality of patient is poor. Because of frequent recurrence and metastasis, clinical efficacy is usually spoiled and prognosis is unsatisfactory. Multi-target treatments may be more effective avenues in treating HCC. TCM has been widely used as a new source of anti-cancer drugs with the advantage of improving the 
adverse reactions in the chemotherapy of cancer. Through the great advance in the finding of active ingredients and in the study of antitumor mechanisms, TCM will give rise to new effective strategies for cancer treatment. TCM has the advantages of less toxic and side effects and more therapeutic targets. Therefore, the combination of TCM and Western medicine is becoming a new trend in the prevention and treatment of liver cancer in modern society. The feasibility and effectiveness of TCM in the treatment of HCC was elucidated in aspects of both clinical evaluations and experimental studies of mechanism in the article. The active ingredients in TCM have been proven to exert antitumor activity through regulation of cancer cell proliferation, metastasis, invasion, cell cycle, autophagy, and apoptosis.

However, the above research has certain limitations, which are mainly manifested in the following aspects. Firstly, some clinical studies contain small sample sizes, and the criteria for inclusion and exclusion are not standardized or mentioned. Some clinical trials take single-center, single-blinded research methods, which could not reflect the therapeutic efficacy in the clinical practice. Secondly, the therapeutic efficiency of TCM varies from person to person due to the different physiques of patients in accordance to the overall concept and characteristics of syndrome differentiation and treatment. Thirdly, the toxic and side effects of certain Chinese medicines on humans and animals are still unclear. Fourthly, in modern medical practice, the effective active ingredients of some Chinese medicines still need to be excavated and verified in pharmacology. In addition, many signal proteins, cytokines and cells existing in the tumor microenvironment affect the progression of liver cancer, while the function of TCM and active ingredients may only be limited in some of them. Owing to its complexity, TCM theory is often questioned; the unknown quality of herbs is a drawback for TCM. Despite these challenges, further well-designed clinical trials and experimental studies will provide promising understanding of the mechanism of TCM and promote the modernization of TCM in the treatment and prevention of HCC.

\section{Abbreviations}

HCC, hepatocellular carcinoma; TCM, traditional Chinese medicine; TACE, transcatheter arterial chemoembolization; JPLQ, Jian Pi Li Qi decoction; PES, postembolization syndromes; CHHJ, Chaihu Huaji decoction; BXXX, Banxia XieXin decoction; PFS, progression-free survival; ESQJR, Erzhu Qinggan Jiedu Recipe; GKR, gamma knife radiosurgery; CCRK, cell-cycle related kinase; AR, androgen receptor; GSK3 $\beta$, glycogen synthase kinase-3 beta; PZH, Pien Tze Huang; ZJP, Zuojin pill; RA, Raddeanin A; ALO, Aloperine; PMT, polygonum multiflorum; CK2, casein kinase 2; GSDMD, gasdermin D; GSDME, gasdermin E; MEK, mitogen-activated and extracellular signal-regulated kinase; ERK1/2, extracellular regulated protein kinases 1/2; CBD, Cannabidiol; ISR, integrative stress response; GPX4, glutathione peroxidase 4; miRNAs, microRNAs; OS, oxidative stress; ROS, reactive oxygen species; AES, $\beta$-Aescin; DOI, Diosmin; DAMPs, damage-associated molecular patterns; DC, dendritic cell; ICD, immunogenic cell death; JLC, Jinlong capsule; FZQJ, Fuzheng Qingjie Granules; FM, Frankincense and myrrh; Treg, regulatory T; APS, Astragalus polysaccharides; MDSCs, Myeloid-derived suppressor cells; JHD, jianpi huayu decoction; TAM, Tumor-associated macrophage; CKI, Compound Kushen Injection; BB, Bombyx batryticatus; GLP, ganoderma lucidum polysaccharide.

\section{Funding}

This work was supported by National Natural Science Foundation of China (No.81874436, 82074154, 81774240), Threeyear Action Plan for the Development of Chinese Medicine in Shanghai (No.ZY (2018-2020)-CCCX-2003-01), Shanghai Key Clinical Specialty Construction Project (No.shslczdzk01201), The Siming Scholar from Shanghai Shuguang Hospital (SGXZ-201904), Youth Tip-top Talent program in Shanghai, Xinglin Youth Scholar from Shanghai University of Traditional Chinese Medicine.

\section{Disclosure}

The authors report no conflicts of interest in this work.

\section{References}

1. Forner A, Reig M, Bruix J. Hepatocellular carcinoma. Lancet. 2018;391(10127):1301-1314.

2. Singal AG, Lampertico P, Nahon P. Epidemiology and surveillance for hepatocellular carcinoma: new trends. J Hepatol. 2020;72(2):250-261. doi:10.1016/j.jhep.2019.08.025 
3. Hartke J, Johnson M, Ghabril M. The diagnosis and treatment of hepatocellular carcinoma. Semin Diagn Pathol. 2017;34(2):153-159. doi:10.1053/ j.semdp.2016.12.011

4. Reddy SK, Steel JL, Chen HW, et al. Outcomes of curative treatment for hepatocellular cancer in nonalcoholic steatohepatitis versus hepatitis C and alcoholic liver disease. Hepatology. 2012;55(6):1809-1819. doi:10.1002/hep.25536

5. Bruix J, Qin S, Merle P, et al. Regorafenib for patients with hepatocellular carcinoma who progressed on sorafenib treatment (RESORCE): a randomised, double-blind, placebo-controlled, Phase 3 trial. Lancet. 2017;389(10064):56-66.

6. Xi SY, Minuk GY. Role of traditional Chinese medicine in the management of patients with hepatocellular carcinoma. World J Hepatol. 2018;10 (11):799-806. doi:10.4254/wjh.v10.i11.799

7. Wang X, Wang N, Cheung F, et al. Chinese medicines for prevention and treatment of human hepatocellular carcinoma: current progress on pharmacological actions and mechanisms. J Integr Med. 2015;13(3):142-164. doi:10.1016/S2095-4964(15)60171-6

8. Sun L, Fahey P, Zhu X, et al. A cohort study to examine the use of Chinese herbal medicine in combination with conventional therapies for patients with hepatocellular carcinoma in China. Integr Cancer Ther. 2018;17(3):902-911. doi:10.1177/1534735418775819

9. Zhai XF, Liu XL, Shen F, et al. Traditional herbal medicine prevents postoperative recurrence of small hepatocellular carcinoma: a randomized controlled study. Cancer. 2018;124(10):2161-2168.

10. Wu T, Chen W, Liu S, et al. Huaier suppresses proliferation and induces apoptosis in human pulmonary cancer cells via upregulation of miR-26b5p. FEBS Lett. 2014;588(12):2107-2114. doi:10.1016/j.febslet.2014.04.044

11. Yan X, Lyu T, Jia N, et al. Huaier aqueous extract inhibits ovarian cancer cell motility via the AKT/GSK3beta/beta-catenin pathway. PLoS One. 2013;8(5):e63731. doi:10.1371/journal.pone.0063731

12. Wang X, Zhang N, Huo Q, et al. Anti-angiogenic and antitumor activities of Huaier aqueous extract. Oncol Rep. 2012;28(4):1167-1175. doi:10.3892/or.2012.1961

13. Song X, Li Y, Zhang H, et al. The anticancer effect of Huaier (Review). Oncol Rep. 2015;34(1):12-21. doi:10.3892/or.2015.3950

14. Chen Q, Shu C, Laurence AD, et al. Effect of Huaier granule on recurrence after curative resection of HCC: a multicentre, randomised clinical trial. Gut. 2018;67(11):2006-2016. doi:10.1136/gutjnl-2018-315983

15. Cheung F, Wang X, Wang N, et al. Chinese medicines as an adjuvant therapy for unresectable hepatocellular carcinoma during transarterial chemoembolization: a meta-analysis of randomized controlled trials. Evid Based Complement Alternat Med. 2013;2013:487919.

16. Zhao GS, Liu Y, Zhang Q, et al. Transarterial chemoembolization combined with Huaier granule for the treatment of primary hepatic carcinoma: safety and efficacy. Medicine (Baltimore). 2017;96(29):e7589. doi:10.1097/MD.0000000000007589

17. Xu L, Wang S, Zhuang L, et al. Jian Pi Li Qi decoction alleviated postembolization syndrome following transcatheter arterial chemoembolization for hepatocellular carcinoma: a randomized, double-blind, placebo-controlled trial. Integr Cancer Ther. 2016;15(3):349-357. doi:10.1177/1534735415617020

18. Shimizu I. Sho-saiko-to: Japanese herbal medicine for protection against hepatic fibrosis and carcinoma. J Gastroenterol Hepatol. 2000;15(Suppl): D84-90. doi:10.1046/j.1440-1746.2000.02138.x

19. Xu H, Deng Y, Zhou Z, et al. Chinese herbal medicine (Chaihu-Huaji Decoction) alleviates postembolization syndrome following transcatheter arterial chemoembolization and improves survival in unresectable hepatocellular cancer: a retrospective study. Evid Based Complement Alternat Med. 2019;2019:6269518. doi:10.1155/2019/6269518

20. Wang L, Ke J, Wang C, et al. Efficacy and safety of Banxia XieXin decoction, a blended Traditional Chinese Medicine, as monotherapy for patients with advanced hepatocellular carcinoma. Integr Cancer Ther. 2020;19:1534735420942587.

21. Cheng Y, Ni S, Chen Y, et al. Erzhu Qinggan Jiedu Recipe improves the clinical outcome of hepatocellular cancer after surgical resection: a case-control retrospective study. Intern Med J. 2021;51(6):853-860. doi:10.1111/imj.14844

22. Shim JS, Kim JH, Lee J, et al. Anti-angiogenic activity of a homoisoflavanone from Cremastra appendiculata. Planta Med. 2004;70(2):171-173.

23. Chen LY, Zhai XF, Chen Z, et al. Jie-du granule preparation for the treatment of advanced hepatocellular carcinoma: a retrospective cohort study of 177 patients. Oncotarget. 2017;8(18):30471-30476. doi:10.18632/oncotarget.12887

24. Wang J, Luo J, Yin X, et al. Jiedu granule combined with transcatheter arterial chemoembolization and gamma knife radiosurgery in treating hepatocellular carcinoma with portal vein tumor thrombus. Biomed Res Int. 2019;2019:4696843.

25. Yu Z, Feng H, Sun X, et al. Bufalin suppresses hepatocarcinogenesis by targeting beta-catenin/TCF signaling via cell cycle-related kinase. Sci Rep. 2018;8(1):3891. doi:10.1038/s41598-018-22113-2

26. Yu Z, Feng H, Zhuo Y, et al. Bufalin inhibits hepatitis B virus-associated hepatocellular carcinoma development through androgen receptor dephosphorylation and cell cycle-related kinase degradation. Cell Oncol (Dordr). 2020;43(6):1129-1145. doi:10.1007/s13402-020-00546-0

27. Hong M, Almutairi MM, Li S, et al. Wogonin inhibits cell cycle progression by activating the glycogen synthase kinase-3 beta in hepatocellular carcinoma. Phytomedicine. 2020;68:153174. doi:10.1016/j.phymed.2020.153174

28. Xu W, Zhang Y, Zhou C, et al. Simultaneous quantification six active compounds in rat plasma by UPLC-MS/MS and its application to a pharmacokinetic study of Pien-Tze-Huang. J Chromatogr B Analyt Technol Biomed Life Sci. 2017;1061-1062:314-321. doi:10.1016/j.jchromb.2017.07.033

29. Fan D, Liu C, Li L, et al. Deciphering antitumor mechanism of Pien Tze Huang in mice of hepatocellular carcinoma based on proteomics. J Immunol Res. 2020;2020:4876251. doi:10.1155/2020/4876251

30. Chou ST, Hsiang CY, Lo HY, et al. Exploration of anti-cancer effects and mechanisms of Zuo-Jin-Wan and its alkaloid components in vitro and in orthotopic HepG2 xenograft immunocompetent mice. BMC Complement Altern Med. 2017;17(1):121.

31. Guo W, Huang J, Wang N, et al. Integrating network pharmacology and pharmacological evaluation for deciphering the action mechanism of herbal formula Zuojin Pill in suppressing hepatocellular carcinoma. Front Pharmacol. 2019;10:1185. doi:10.3389/fphar.2019.01185

32. Zhang B, Yin X, Sui S. Resveratrol inhibited the progression of human hepatocellular carcinoma by inducing autophagy via regulating p53 and the phosphoinositide 3kinase/protein kinase B pathway. Oncol Rep. 2018;40(5):2758-2765.

33. Dai H, Li M, Yang W, et al. Resveratrol inhibits the malignant progression of hepatocellular carcinoma via MARCH1-induced regulation of PTEN/ AKT signaling. Aging (Albany NY). 2020;12(12):11717-11731. doi:10.18632/aging.103338

34. Elledge SJ. Cell cycle checkpoints: preventing an identity crisis. Science. 1996;274(5293):1664-1672. doi:10.1126/science.274.5293.1664

35. Li JN, Yu Y, Zhang YF, et al. Synergy of Raddeanin A and cisplatin induced therapeutic effect enhancement in human hepatocellular carcinoma. Biochem Biophys Res Commun. 2017;485(2):335-341. doi:10.1016/j.bbrc.2017.02.079

36. Lin ZK, Liu J, Jiang GQ, et al. Osthole inhibits the tumorigenesis of hepatocellular carcinoma cells. Oncol Rep. 2017;37(3):1611-1618. doi:10.3892/or.2017.5403 
37. Liu J, Huo C, Cao H, et al. Aloperine induces apoptosis and G2/M cell cycle arrest in hepatocellular carcinoma cells through the PI3K/Akt signaling pathway. Phytomedicine. 2019;61:152843. doi:10.1016/j.phymed.2019.152843

38. Elmore S. Apoptosis: a review of programmed cell death. Toxicol Pathol. 2007;35(4):495-516. doi:10.1080/01926230701320337

39. Fernald K, Kurokawa M. Evading apoptosis in cancer. Trends Cell Biol. 2013;23(12):620-633.

40. Kossler S, Nofziger C, Jakab M, et al. Curcumin affects cell survival and cell volume regulation in human renal and intestinal cells. Toxicology. 2012;292(2-3):123-135. doi:10.1016/j.tox.2011.12.002

41. Zeng Y, Shen Z, Gu W, et al. Inhibition of hepatocellular carcinoma tumorigenesis by curcumin may be associated with CDKN1A and CTGF. Gene. 2018;651:183-193. doi:10.1016/j.gene.2018.01.083

42. Kun-Ming C, Chih-Hsien C, Chen-Fang L, et al. Potential anticancer effect of celastrol on hepatocellular carcinoma by suppressing CXCR4-related signal and impeding tumor growth in vivo. Arch Med Res. 2020;51(4):297-302. doi:10.1016/j.arcmed.2020.03.001

43. Rajendran P, Li F, Shanmugam MK, et al. Celastrol suppresses growth and induces apoptosis of human hepatocellular carcinoma through the modulation of STAT3/JAK2 signaling cascade in vitro and in vivo. Cancer Prev Res (Phila). 2012;5(4):631-643. doi:10.1158/1940-6207.CAPR-11-0420

44. Li J, Liu P, Zhang R, et al. Icaritin induces cell death in activated hepatic stellate cells through mitochondrial activated apoptosis and ameliorates the development of liver fibrosis in rats. J Ethnopharmacol. 2011;137(1):714-723.

45. Yu Z, Guo J, Hu M, et al. Icaritin exacerbates mitophagy and synergizes with doxorubicin to induce immunogenic cell death in hepatocellular carcinoma. ACS Nano. 2020;14(4):4816-4828. doi:10.1021/acsnano.0c00708

46. Xue X, Quan Y, Gong L, et al. A review of the processed Polygonum multiflorum (Thunb.) for hepatoprotection: clinical use, pharmacology and toxicology. J Ethnopharmacol. 2020;261:113121. doi:10.1016/j.jep.2020.113121

47. Wang H, Xiao Y, Wang H, et al. Development of daidzein nanosuspensions: preparation, characterization, in vitro evaluation, and pharmacokinetic analysis. Int J Pharm. 2019;566:67-76. doi:10.1016/j.ijpharm.2019.05.051

48. Kim HR, Kim K, Lee KH, et al. Inhibition of casein kinase 2 enhances the death ligand- and natural killer cell-induced hepatocellular carcinoma cell death. Clin Exp Immunol. 2008;152(2):336-344. doi:10.1111/j.1365-2249.2008.03622.x

49. Bai J, Wu J, Tang R, et al. Emodin, a natural anthraquinone, suppresses liver cancer in vitro and in vivo by regulating VEGFR(2) and miR-34a. Invest New Drugs. 2020;38(2):229-245. doi:10.1007/s10637-019-00777-5

50. Pan XP, Wang C, Li Y, et al. Physcion induces apoptosis through triggering endoplasmic reticulum stress in hepatocellular carcinoma. Biomed Pharmacother. 2018;99:894-903. doi:10.1016/j.biopha.2018.01.148

51. Pan X, Wang C, Zhang T. Physcion synergistically enhances the cytotoxicity of sorafenib in hepatocellular carcinoma. Anat Rec (Hoboken). 2019;302(12):2171-2177. doi:10.1002/ar.24179

52. Wang Y, Gao W, Shi X, et al. Chemotherapy drugs induce pyroptosis through caspase-3 cleavage of a gasdermin. Nature. 2017;547(7661):99-103. doi:10.1038/nature22393

53. Chen X, He WT, Hu L, et al. Pyroptosis is driven by non-selective gasdermin-D pore and its morphology is different from MLKL channel-mediated necroptosis. Cell Res. 2016;26(9):1007-1020. doi:10.1038/cr.2016.100

54. Fang Y, Tian S, Pan Y, et al. Pyroptosis: a new frontier in cancer. Biomed Pharmacother. 2020;121:109595. doi:10.1016/j.biopha.2019.109595

55. Zhang X, Zhang P, An L, et al. Miltirone induces cell death in hepatocellular carcinoma cell through GSDME-dependent pyroptosis. Acta Pharm Sin B. 2020;10(8):1397-1413. doi:10.1016/j.apsb.2020.06.015

56. Shangguan F, Zhou H, Ma N, et al. A novel mechanism of cannabidiol in suppressing hepatocellular carcinoma by inducing GSDME dependent pyroptosis. Front Cell Dev Biol. 2021;9:697832. doi:10.3389/fcell.2021.697832

57. Kis B, Ifrim FC, Buda V, et al. Cannabidiol-from plant to human body: a promising bioactive molecule with multi-target effects in cancer. Int $J$ Mol Sci. 2019;20:23. doi:10.3390/ijms20235905

58. Hadian K, Stockwell BR. SnapShot: ferroptosis. Cell. 2020;181(5):1188-1188.e1181. doi:10.1016/j.cell.2020.04.039

59. Stockwell BR, Friedmann Angeli JP, Bayir H, et al. Ferroptosis: a regulated cell death nexus linking metabolism, redox biology, and disease. Cell. 2017;171(2):273-285. doi:10.1016/j.cell.2017.09.021

60. Dixon SJ, Lemberg KM, Lamprecht MR, et al. Ferroptosis: an iron-dependent form of nonapoptotic cell death. Cell. 2012;149(5):1060-1072. doi:10.1016/j.cell.2012.03.042

61. Fearnhead HO, Vandenabeele P, Vanden Berghe T. How do we fit ferroptosis in the family of regulated cell death? Cell Death Differ. 2017;24 (12):1991-1998. doi:10.1038/cdd.2017.149

62. Mou Y, Wang J, Wu J, et al. Ferroptosis, a new form of cell death: opportunities and challenges in cancer. J Hematol Oncol. 2019;12(1):34. doi:10.1186/s13045-019-0720-y

63. Kotawong K, Chaijaroenkul W, Muhamad P, et al. Cytotoxic activities and effects of atractylodin and $\beta$-eudesmol on the cell cycle arrest and apoptosis on cholangiocarcinoma cell line. J Pharmacol Sci. 2018;136(2):51-56. doi:10.1016/j.jphs.2017.09.033

64. He Y, Fang D, Liang T, et al. Atractylodin may induce ferroptosis of human hepatocellular carcinoma cells. Ann Transl Med. 2021;9(20):1535. doi:10.21037/atm-21-4386

65. Shen KH, Hung JH, Chang CW, et al. Solasodine inhibits invasion of human lung cancer cell through downregulation of miR-21 and MMPs expression. Chem Biol Interact. 2017;268:129-135. doi:10.1016/j.cbi.2017.03.005

66. Xu XH, Zhang LL, Wu GS, et al. Solasodine induces apoptosis, affects autophagy, and attenuates metastasis in ovarian cancer cells. Planta Med. 2017;83(3-4):254-260.

67. Jin M, Shi C, Li T, et al. Solasonine promotes ferroptosis of hepatoma carcinoma cells via glutathione peroxidase 4-induced destruction of the glutathione redox system. Biomed Pharmacother. 2020;129:110282. doi:10.1016/j.biopha.2020.110282

68. Chen K, Zhang S, Ji Y, et al. Baicalein inhibits the invasion and metastatic capabilities of hepatocellular carcinoma cells via down-regulation of the ERK pathway. PLoS One. 2013;8(9):e72927. doi:10.1371/journal.pone.0072927

69. Liang RR, Zhang S, Qi JA, et al. Preferential inhibition of hepatocellular carcinoma by the flavonoid Baicalein through blocking MEK-ERK signaling. Int J Oncol. 2012;41(3):969-978. doi:10.3892/ijo.2012.1510

70. Min R, Tong J, Wenjun Y, et al. Growth inhibition and induction of apoptosis in human oral squamous cell carcinoma Tca-8113 cell lines by Shikonin was partly through the inactivation of NF-kappaB pathway. Phytother Res. 2008;22(3):407-415. doi:10.1002/ptr.2340 
71. Hsu PC, Huang YT, Tsai ML, et al. Induction of apoptosis by shikonin through coordinative modulation of the Bcl-2 family, p27, and p53, release of cytochrome c, and sequential activation of caspases in human colorectal carcinoma cells. J Agric Food Chem. 2004;52(20):6330-6337. doi:10.1021/jf0495993

72. Gong K, Zhang Z, Chen Y, et al. Extracellular signal-regulated kinase, receptor interacting protein, and reactive oxygen species regulate shikonin-induced autophagy in human hepatocellular carcinoma. Eur J Pharmacol. 2014;738:142-152. doi:10.1016/j.ejphar.2014.05.034

73. Amin A, Hamza AA, Bajbouj K, et al. Saffron: a potential candidate for a novel anticancer drug against hepatocellular carcinoma. Hepatology. 2011;54(3):857-867. doi:10.1002/hep.24433

74. Yao C, Liu B, Qian X, et al. Crocin induces autophagic apoptosis in hepatocellular carcinoma by inhibiting Akt/mTOR activity. Onco Targets Ther. 2018;11:2017-2028. doi:10.2147/OTT.S154586

75. Juaid N, Amin A, Abdalla A, et al. Anti-hepatocellular carcinoma biomolecules: molecular targets insights. Int J Mol Sci. 2021;22(19):10774. doi:10.3390/ijms221910774

76. Jiang N, Xin W, Wang T, et al. Protective effect of aescin from the seeds of Aesculus hippocastanum on liver injury induced by endotoxin in mice. Phytomedicine. 2011;18(14):1276-1284. doi:10.1016/j.phymed.2011.06.011

77. Tahir M, Rehman MU, Lateef A, et al. Diosmin protects against ethanol-induced hepatic injury via alleviation of inflammation and regulation of TNF- $\alpha$ and NF- $\kappa$ B activation. Alcohol. 2013;47(2):131-139. doi:10.1016/j.alcohol.2012.12.010

78. El-Dakhly SM, Salama AAA, Hassanin SOM, et al. Aescin and diosmin each alone or in low dose- combination ameliorate liver damage induced by carbon tetrachloride in rats. BMC Res Notes. 2020;13(1):259. doi:10.1186/s13104-020-05094-2

79. Prasad S, Tyagi AK. Ginger and its constituents: role in prevention and treatment of gastrointestinal cancer. Gastroenterol Res Pract. 2015;2015:142979. doi:10.1155/2015/142979

80. Hamza AA, Heeba GH, Hamza S, et al. Standardized extract of ginger ameliorates liver cancer by reducing proliferation and inducing apoptosis through inhibition oxidative stress/ inflammation pathway. Biomed Pharmacother. 2021;134:111102. doi:10.1016/j.biopha.2020.111102

81. Jiang ZT, Han Y, Liu XY, et al. Tripterine restrains the aggressiveness of hepatocellular carcinoma cell via regulating miRNA-532-5p/CXCL2 axis. Onco Targets Ther. 2020;13:2973-2985. doi:10.2147/OTT.S238074

82. Vishnoi K, Ke R, Saini KS, et al. Berberine represses beta-catenin translation involving 4E-BPs in hepatocellular carcinoma cells. Mol Pharmacol. 2021;99(1):1-16. doi:10.1124/molpharm.120.000029

83. Yu R, Zhang ZQ, Wang B, et al. Berberine-induced apoptotic and autophagic death of HepG2 cells requires AMPK activation. Cancer Cell Int. 2014;14:49. doi:10.1186/1475-2867-14-49

84. Martin D, Galisteo R, Gutkind JS. CXCL8/IL8 stimulates vascular endothelial growth factor (VEGF) expression and the autocrine activation of VEGFR2 in endothelial cells by activating NFkappaB through the CBM (Carma3/Bcl10/Malt1) complex. J Biol Chem. 2009;284(10):6038-6042. doi:10.1074/jbc.C800207200

85. Lin W, Wang H, Zhong M, et al. Effect and molecular mechanisms of Jiedu recipe on hypoxia-induced angiogenesis after transcatheter arterial chemoembolization in hepatocellular carcinoma. Evid Based Complement Alternat Med. 2021;2021:6529376.

86. Zang W, Bian H, Huang X, et al. Traditional Chinese Medicine (TCM) Astragalus Membranaceus and Curcuma Wenyujin promote vascular normalization in tumor-derived endothelial cells of human hepatocellular carcinoma. Anticancer Res. 2019;39(6):2739-2747. doi:10.21873/ anticanres. 13400

87. Xu H, Wei W, Y. M, Dong C. Efficacy and safety of Chinese patent medicine (Jinlong capsule) in the treatment of advanced hepatocellular carcinoma: a meta-analysis. Biosci Rep. 2020;40(1):BSR20194019. doi:10.1042/BSR20194019

88. Chen X, Cao Z, Zhang Y, et al. Fuzheng Qingjie granules inhibit growth of hepatoma cells via inducing mitochondria-mediated apoptosis and enhancing immune function. Integr Cancer Ther. 2017;16(3):329-338. doi:10.1177/1534735416654761

89. Su S, Duan J, Chen T, et al. Frankincense and myrrh suppress inflammation via regulation of the metabolic profiling and the MAPK signaling pathway. Sci Rep. 2015;5:13668. doi:10.1038/srep13668

90. Xu C, Lu X, Liu W, et al. CD8(+) T cells mediate the antitumor activity of frankincense and myrrh in hepatocellular carcinoma. $J$ Transl Med. 2018;16(1):132. doi:10.1186/s12967-018-1508-5

91. Zhang Q, Huang H, Zheng F, et al. Resveratrol exerts antitumor effects by downregulating CD8(+)CD122(+) Tregs in murine hepatocellular carcinoma. Oncoimmunology. 2020;9(1):1829346. doi:10.1080/2162402X.2020.1829346

92. Li Q, Bao JM, Li XL, et al. Inhibiting effect of Astragalus polysaccharides on the functions of CD4+CD25 highTreg cells in the tumor microenvironment of human hepatocellular carcinoma. Chin Med J (Engl). 2012;125(5):786-793.

93. Xie Y, Zhang Y, Wei X, et al. Jianpi Huayu decoction attenuates the immunosuppressive status of H(22) hepatocellular carcinoma-bearing mice: by targeting myeloid-derived suppressor cells. Front Pharmacol. 2020;11:16. doi:10.3389/fphar.2020.00016

94. Yang Y, Sun M, Yao W, et al. Compound kushen injection relieves tumor-associated macrophage-mediated immunosuppression through TNFR1 and sensitizes hepatocellular carcinoma to sorafenib. J Immunother Cancer. 2020;8(1):e000317. doi:10.1136/jitc-2019-000317

95. Yuan L, Bing Z, Han J, et al. Study on the anti-tumor mechanism related to immune microenvironment of Bombyx Batryticatus on viral and non-viral infections of hepatocellular carcinoma. Biomed Pharmacother. 2020;124:109838. doi:10.1016/j.biopha.2020.109838

96. He S, Tian S, He X, et al. Multiple targeted self-emulsifying compound RGO reveals obvious anti-tumor potential in hepatocellular carcinoma. Mol Ther Oncolytics. 2021;22:604-616.

Journal of Hepatocellular Carcinoma

Dovepress

\section{Publish your work in this journal}

The Journal of Hepatocellular Carcinoma is an international, peer-reviewed, open access journal that offers a platform for the dissemination and study of clinical, translational and basic research findings in this rapidly developing field. Development in areas including, but not limited to, epidemiology, vaccination, hepatitis therapy, pathology and molecular tumor classification and prognostication are all considered for publication. The manuscript management system is completely online and includes a very quick and fair peer-review system, which is all easy to use. Visit http://www.dovepress.com/testimonials.php to read real quotes from published authors.

Submit your manuscript here: https://www.dovepress.com/journal-of-hepatocellular-carcinoma-journal 